\title{
Verification of the Observer Property in Discrete Event Systems
}

\author{
P. N. Pena, H. J. Bravo, A. E. C. da Cunha, R. Malik, \\ S. Lafortune, Fellow, IEEE, and J. E. R. Cury, Member, IEEE
}

\begin{abstract}
The observer property is an important condition to be satisfied by abstractions of Discrete Event System (DES) models. This paper presents a new algorithm that tests if an abstraction of a DES obtained through natural projection has the observer property. The procedure, called OP-Verifier, can be applied to (potentially nondeterministic) automata, with no restriction on the existence of cycles of "non-relevant" events. This procedure has quadratic complexity in the number of states. The performance of the algorithm is illustrated by a set of experiments.
\end{abstract}

Index Terms-Discrete Event Systems, Natural Projections, Observer Property.

\section{INTRODUCTION}

Natural projections play a central role in the computation of abstractions for Discrete Event Systems (DES) models. Abstractions obtained by natural projections have been extensively used in the Supervisory Control Theory of DES [1] as, for example, in control with partial observation, in hierarchical control [2]-[6], in modular synthesis [2], [7], [8], and in compositional verification of the nonblocking property [2], [9], [10], among many problem domains. In several of the above cited works, the observer property is an important condition to be satisfied by the abstracted models. Abstractions satisfying this property are called OP-abstractions [11].

The observer property, or simply OP hereafter, was first introduced in the context of hierarchical control of DES In [12], the abstraction is obtained in the form of a reporter map, that projects strings of events of the original (low-level) model, built from a set $\Sigma$, into high-level strings built from an independent set of events, denoted by $T$. Due to some difficulties with the use of reporter maps [13], most of the approaches subsequent to [12] focus on abstractions obtained by the natural projection, which maps strings of the original model into strings of the abstraction, by erasing events of $\Sigma$

P. N. Pena is with the Departamento de Engenharia Eletrônica, Universidade Federal de Minas Gerais, Brazil (e-mail: ppena@ufmg.br).

H. J. Bravo and A. E. C. da Cunha are with the Seção de Engenharia Elétrica, Instituto Militar de Engenharia, Brazil (email: hugobravoc@gmail.com, carrilho@ime.eb.br).

R. Malik is with the Department of Computer Science, The University of Waikato, New Zealand (e-mail: robi@waikato.ac.nz).

S. Lafortune is with the Department of Electrical Engineering and Computer Science, The University of Michigan, USA (e-mail: stephane@eecs.umich.edu).

J. E. R. Cury is with the Departamento de Automação e Sistemas, Universidade Federal de Santa Catarina, Brazil (e-mail: jose.cury@ufsc.br).

The first, second, third, and sixth authors are supported in part by CAPES (PROCAD 102/2007). The first and sixth authors are supported in part by FAPEMIG and CNPq grant 300953/93-3, respectively. The research of the fifth author is supported in part by NSF grant CNS-0930081. that are not contained in a given subset of relevant events, denoted by $\Sigma_{r}$, with $\Sigma_{r} \subseteq \Sigma$; see [3]-[5], [14], [15].

The structure called $O P$-Verifier was first presented in [11]; it was inspired by an algorithm for testing diagnosability presented in [16]. Given an input automaton $G$, defined on the alphabet $\Sigma$, a set of relevant events $\Sigma_{r} \subseteq \Sigma$, and a natural projection $\theta$ from strings in $\Sigma$ to strings in $\Sigma_{r}$, the $O P$ Verifier algorithm checks whether the projection $\theta\left(\mathcal{L}_{m}(G)\right)$ is an $O P$-abstraction. The $O P$-Verifier algorithm does not require explicitly computing the abstraction to check for the OP and has been shown to have better computational performance when compared to other similar procedures [13], [17]-[19]. It runs in quadratic complexity in the number of states. A limitation of the $O P$-Verifier algorithm as proposed in [11], however, is that it can only be applied to automata that do not have cycles of non-relevant events.

A different algorithm to test the OP is proposed in [13], [17]. This algorithm relies on the computation of a coarsest observation equivalence relation and runs in cubic complexity in the number of states. Yet another algorithm for testing so-called "observerness" for a system $G$ and a mask $M$ is presented in [18]. This procedure may give false negatives as stated and needs to be modified to address this problem [20].

This paper presents a modified version of the OP-Verifier algorithm of [11] that subsumes the preliminary results in [21]. This algorithm can be applied to automata with no restriction on the existence of cycles of non-relevant events. The algorithm operates on a modified automaton $G_{\mathrm{nr}}$, obtained from the input automaton $G$, by aggregating states connected by cycles of non-relevant events. It overcomes the limitations of the previously proposed verifier [11], [21], while retaining its quadratic complexity. The modified $O P$-Verifier algorithm has been implemented in Supremica [22].

This paper is organized as follows. Section II introduces the necessary background. Section III describes the construction of the OP-Verifier automaton and its properties. Then Section IV presents an algorithm to construct the OP-Verifier and check the observer property. This section also contains a complexity analysis and experimental results to demonstrate the performance of the algorithm in comparison with [17]. Finally, concluding remarks are given in Section V.

\section{Preliminaries}

This paper is set in the supervisory control framework. The reader is referred to [1] for a detailed introduction to the theory. Behaviors of DES are modeled using strings of events 
taken from a finite alphabet $\Sigma . \Sigma^{*}$ is the set of all finite strings of events in $\Sigma$, including the empty string $\varepsilon$. The concatenation of strings $s, u \in \Sigma^{*}$ is written as $s u$. A string $s \in \Sigma^{*}$ is called a prefix of $t \in \Sigma^{*}$, written $s \leq t$, if there exists $u \in \Sigma^{*}$ such that $s u=t$. A subset $L \subseteq \Sigma^{*}$ is called a language. The prefixclosure $\bar{L}$ of a language $L \subseteq \Sigma^{*}$ is the set of all prefixes of strings in $L$, i.e., $\bar{L}=\left\{s \in \Sigma^{*} \mid s \leq t\right.$ for some $\left.t \in L\right\}$. Regular languages are represented by (possibly nondeterministic) finite-state automata as follows.

Definition 1: A (nondeterministic) finite-state automaton is a tuple $G=\left\langle\Sigma, Q, \rightarrow, Q^{\circ}\right\rangle$, where $\Sigma$ is a finite set of events, $Q$ is a finite set of states, $\rightarrow \subseteq Q \times \Sigma \times Q$ is the state transition relation, and $Q^{\circ} \subseteq Q$ is the set of initial states. $G$ is deterministic, if $\left|Q^{\circ}\right| \leq 1$ and $x \stackrel{\sigma}{\rightarrow} y_{1}$ and $x \stackrel{\sigma}{\rightarrow} y_{2}$ always implies $y_{1}=y_{2}$.

The transition relation is written in infix notation $x \stackrel{\sigma}{\rightarrow} y$, and is extended to traces in $\Sigma^{*}$ by letting $x \stackrel{\varepsilon}{\rightarrow} x$ for all $x \in Q$, and $x \stackrel{s \sigma}{\rightarrow} z$ if $x \stackrel{s}{\rightarrow} y$ and $y \stackrel{\sigma}{\rightarrow} z$ for some $y \in Q$. Furthermore, $x \stackrel{s}{\rightarrow}$ means $x \stackrel{s}{\rightarrow} y$ for some $y \in Q$, and $x \rightarrow y$ means $x \stackrel{s}{\rightarrow} y$ for some $s \in \Sigma^{*}$. These notations also apply to state sets: $X \stackrel{s}{\rightarrow} Y$ for $X, Y \subseteq Q$ means $x \stackrel{s}{\rightarrow} y$ for some states $x \in X$ and $y \in Y$. Also, if $G$ is an automaton, then $G \stackrel{s}{\rightarrow} x, G \stackrel{s}{\rightarrow} X$, and $G \stackrel{s}{\rightarrow}$ stand for $Q^{\circ} \stackrel{s}{\rightarrow} x, Q^{\circ} \stackrel{s}{\rightarrow} X$, and $Q^{\circ} \stackrel{s}{\rightarrow}$, respectively. For example, $G \stackrel{s}{\rightarrow} X$ means that the automaton $G$ can reach some state in the set $X \subseteq Q$ on execution of trace $s \in \Sigma^{*}$. Finally, the generated language of automaton $G$ is $\mathcal{L}(G)=\left\{s \in \Sigma^{*} \mid G \stackrel{s}{\rightarrow}\right\}$.

To express the marking of strings, the alphabet $\Sigma$ is assumed to contain the marking event $\omega \in \Sigma$, which may only appear on self-loops, i.e., $x \stackrel{\omega}{\rightarrow} y$ always implies $x=y$. In this notation, the marked language of $G$ is defined as $\mathcal{L}_{\mathrm{m}}(G)=\left\{s \in(\Sigma \backslash\{\omega\})^{*} \mid s \omega \in \mathcal{L}(G)\right\}$. This paper uses the marking event $\omega$ instead of the more conventional set of marking, or final, states, because it simplifies the presentation by associating the marking of strings to a special case of transition.

Given an automaton $G=\left\langle\Sigma, Q, \rightarrow, Q^{\circ}\right\rangle$, a state $x \in Q$ is called reachable if $G \rightarrow x$, and coreachable if $x \stackrel{t \omega}{\longrightarrow}$ for some $t \in \Sigma^{*}$. The automaton $G$ is called reachable if every state $x \in Q$ is reachable, and nonblocking if every reachable state $x \in Q$ is coreachable.

A common automaton operation is the quotient modulo an equivalence relation on the state set.

Definition 2: Let $G=\left\langle\Sigma, Q, \rightarrow, Q^{\circ}\right\rangle$ be an automaton and let $\sim \subseteq Q \times Q$ be an equivalence relation. The quotient automaton of $G$ modulo $\sim$ is

$$
G / \sim=\left\langle\Sigma, Q / \sim, \rightarrow / \sim, \tilde{Q}^{\circ}\right\rangle,
$$

where $\rightarrow / \sim=\left\{([x], \sigma,[y]) \mid x^{\prime} \stackrel{\sigma}{\rightarrow} y^{\prime}\right.$ for some $x^{\prime} \in[x]$ and $\left.y^{\prime} \in[y]\right\}$ and $\tilde{Q}^{\circ}=\left\{\left[x^{\circ}\right] \mid x^{\circ} \in Q^{\circ}\right\}$. Here, $[x]=\left\{x^{\prime} \in\right.$ $\left.Q \mid x \sim x^{\prime}\right\}$ denotes the equivalence class of $x \in Q$, and $Q / \sim=\{[x] \mid x \in Q\}$ is the set of all equivalence classes.

An operation over languages that is very important for abstraction is natural projection. For this purpose, the event alphabet is partitioned into $\Sigma=\Sigma_{\mathrm{r}} \dot{\cup} \Sigma_{\mathrm{nr}}$, where $\Sigma_{\mathrm{r}}$ denotes the set of relevant events, while $\Sigma_{\mathrm{nr}}$ denotes the set of nonrelevant events. For $\Sigma_{\mathrm{r}} \subseteq \Sigma$, the natural projection $\theta: \Sigma^{*} \rightarrow$ $\Sigma_{\mathrm{r}}^{*}$ maps strings in $\Sigma^{*}$ to strings in $\Sigma_{\mathrm{r}}^{*}$ by erasing all events
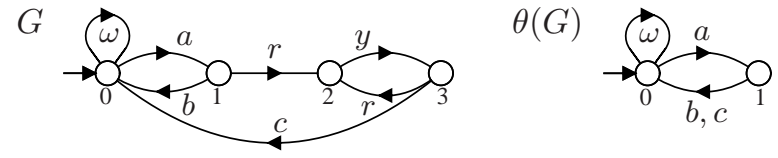

Fig. 1. Example to demonstrate the observer property.

not contained in $\Sigma_{\mathrm{r}}$. The concept is extended to languages by defining $\theta(L)=\left\{t \in \Sigma_{\mathrm{r}}^{*} \mid t=\theta(s)\right.$ for some $\left.s \in L\right\}$.

This paper is concerned with the property of projections known as the observer property, which was first introduced in the context of reporter maps in [12] and [14]. In the context of natural projections, it is written as follows.

Definition 3: [14] Let $L \subseteq \Sigma^{*}$ be a language, let $\Sigma_{\mathrm{r}} \subseteq \Sigma$, and let $\theta: \Sigma^{*} \rightarrow \Sigma_{\mathrm{r}}^{*}$ be the natural projection. If for all $s \in \bar{L}$ and all $t \in \Sigma_{\mathrm{r}}^{*}$ such that $\theta(s) t \in \theta(L)$, there exists $t^{\prime} \in \Sigma^{*}$ such that $\theta\left(s t^{\prime}\right)=\theta(s) t$ and $s t^{\prime} \in L$, then $\theta(L)$ has the observer property.

The observer property ensures that, if two states can be reached by traces with the same projection, i.e., $G \stackrel{s_{1}}{\rightarrow} x_{1}$ and $G \stackrel{s_{2}}{\rightarrow} x_{2}$ with $\theta\left(s_{1}\right)=\theta\left(s_{2}\right)$, then these states can also achieve termination by traces with equal projection, i.e., $x_{1} \stackrel{t_{1} \omega}{\longrightarrow}$ implies $x_{2} \stackrel{t_{2} \omega}{\longrightarrow}$ with $\theta\left(t_{1}\right)=\theta\left(t_{2}\right)$. If the observer property is satisfied for an automaton, then its natural projection is "observation equivalent" to that automaton, which means that all branching in the automaton remains visible in its projection [12].

Projections can also be applied to automata. Given a deterministic and nonblocking automaton $G$, its projection $\theta(G)$ is the minimal deterministic recognizer of the language $\theta\left(\mathcal{L}_{\mathrm{m}}(G)\right)$ [23]. Then it is said that $\theta(G)$ has the observer property if $\theta\left(\mathcal{L}_{\mathrm{m}}(G)\right)$ has the observer property. In this case $\theta(G)$ is also called an $O P$-abstraction.

Example 1: Automaton $G$ in Fig. 1 models the behavior of a simple manufacturing transfer line with material feedback, adapted from [21], [24]. After starting to manufacture a workpiece $(a)$, the transfer line can either finish production sucessfully $(b)$, or decide to retain the workpiece $(r)$ for one or more rework cycles $(y)$, and eventually finish production with a reworked workpiece $(c)$. Assume that, in some hierarchical control approach, as in [3], [5], [6], one is concerned only with the input-output behavior of the line. Then it is of interest to construct the abstraction $\theta(G)$ with respect to relevant events $\Sigma_{\mathrm{r}}=\{a, b, c, \omega\}$ and non-relevant events $\Sigma_{\mathrm{nr}}=\{r, y\}$, which is shown in Fig. 1. In this case, $\theta(G)$ is not an OP-abstraction. To see this, let $s=a r$ and $t=b$ in Definition 3. Then $\theta(s) t=a b \in \theta\left(\mathcal{L}_{\mathrm{m}}(G)\right)$, but there is no trace $t^{\prime} \in \Sigma^{*}$ such that $s t^{\prime}=a r t^{\prime} \in \mathcal{L}_{\mathrm{m}}(G)$ and $\theta\left(s t^{\prime}\right)=\theta(s) t=a b$.

The $O P$-Verifier algorithm [11] can check for certain projections whether or not they satisfy the observer property. This algorithm, which was inspired by the verifier [16] for testing the property of diagnosability, can only be applied to deterministic automata that do not have cycles of non-relevant events. The automaton $G$ in Fig. 1 has a cycle of non-relevant events involving states 1,2 , and 3 . Because of this cycle, the example cannot be classified correctly by the algorithm [11]. 


\section{VERIFICATION OF THE OBSERVER PROPERTY}

In this section, the OP-Verifier algorithm is presented. It extends the algorithm in [11] by adding the ability to handle cycles of non-relevant events.

\section{A. Strongly $\Sigma_{\mathrm{nr}}$-Connected Components Automaton $G_{\mathrm{nr}}$}

In order to deal with cycles of non-relevant events, a strongly $\Sigma_{\mathrm{nr}}$-connected components automaton is introduced. Let $G=\left\langle\Sigma, Q, \rightarrow, Q^{\circ}\right\rangle$ be an automaton, and let $\Sigma_{\mathrm{nr}} \subseteq \Sigma$ be a set of non-relevant events. Define the following relations on the state set $Q$ :

$$
\begin{aligned}
& x \stackrel{\mathrm{nr}}{\longrightarrow} y \Longleftrightarrow x \stackrel{s}{\longrightarrow} y \text { for some } s \in \Sigma_{\mathrm{nr}}^{*} ; \\
& x \stackrel{\mathrm{nr}}{\longleftrightarrow} y \Longleftrightarrow x \stackrel{\mathrm{nr}}{\longrightarrow} y \text { and } y \stackrel{\mathrm{nr}}{\longrightarrow} x .
\end{aligned}
$$

If $x \stackrel{\mathrm{nr}}{\leftrightarrow} y$, then the states $x$ and $y$ are called strongly $\Sigma_{\mathrm{nr}}$ connected $\left(\Sigma_{\mathrm{nr}}-\mathrm{SC}\right)$, because it is possible to reach each state from the other using only non-relevant events. If $\mathrm{G}$ does not contain two distinct $\Sigma_{\mathrm{nr}}$-SC states it is said to be $\Sigma_{\mathrm{nr}}$-acyclic.

A set of $\Sigma_{\mathrm{nr}}$-SC states is called a strongly $\Sigma_{\mathrm{nr}}$-connected component $\left(\Sigma_{\mathrm{nr}}-\mathrm{SCC}\right)$. If each $\Sigma_{\mathrm{nr}}-\mathrm{SCC}$ is contracted to a single state, the resulting automaton is $\Sigma_{n r}$-acyclic. This contracted automaton is called the strongly $\Sigma_{\mathrm{nr}}$-connected components automaton ( $\Sigma_{\mathrm{nr}} \mathrm{SCC}$ automaton) of $G$ in the following. Formally, the $\Sigma_{\mathrm{nr}}$-SCC of state $x \in Q$ is

$$
[x]=\{y \in Q \mid x \stackrel{\mathrm{nr}}{\leftrightarrow} y\},
$$

and the $\Sigma_{\mathrm{nr}}$-SCC automaton of $G$ is the quotient automaton constructed by merging the $\Sigma_{\mathrm{nr}}$-SCCs in $G$,

$$
G_{\mathrm{nr}}=G / \stackrel{\mathrm{nr}}{\leftrightarrow} .
$$

Remark 1: In graph theory, the $\Sigma_{\mathrm{nr}}-\mathrm{SCC}$ automaton is called a condensation graph, which is known to be acyclic [25], i.e., it does not contain any cycles of non-relevant events except for self-loops. For a finite state set, it follows that for every state $x \in Q$, there exists a state $y \in Q$ such that $x \stackrel{\mathrm{nr}}{\longrightarrow} y$, with $[y]$ a terminal component, i.e., a component with no further $\Sigma_{\mathrm{nr}}$-transitions outgoing to other components.

Definition 4: Let $G=\left\langle\Sigma, Q, \rightarrow, Q^{\circ}\right\rangle$ be an automaton, and let $\Sigma_{\mathrm{nr}} \subseteq \Sigma$. For $y \in Q$, the component $[y]$ is $\Sigma_{\mathrm{nr}}$-terminal if, for all $\sigma \in \Sigma_{\mathrm{nr}}$ and all $z \in Q$ such that $[y] \stackrel{\sigma}{\rightarrow}[z]$, it holds that $[y]=[z]$.

The strongly connected components of a graph can be computed efficiently using Tarjan's Algorithm [26]. This algorithm has a worst-case time complexity of $O(|\rightarrow|)$, i.e., it is linear in the number of transitions. Tarjan's Algorithm can be easily adapted to compute the $\Sigma_{n r}$-SCC automaton.

\section{B. OP-Verifier $V_{G}$}

Based on the $\Sigma_{\mathrm{nr}}$-SCC automaton, the OP-Verifier $V_{G}$ is constructed. The $O P$-Verifier is a nondeterministic automaton that is used to determine whether or not the observer property is satisfied for the original automaton $G$ and non-relevant events $\Sigma_{\mathrm{nr}}$. It is constructed in a similar way to the previous $O P$-Verifier for $\Sigma_{\mathrm{nr}}$-acyclic automata in [11], except that it is based on the $\Sigma_{\mathrm{nr}}$-SCC automaton $G_{\mathrm{nr}}$ instead of $G$.
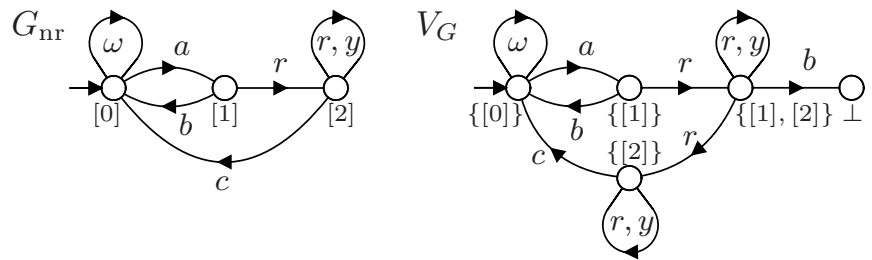

Fig. 2. Example of OP-Verifier construction.

Definition 5: Let $G=\left\langle\Sigma, Q, \rightarrow, Q^{\circ}\right\rangle$ be a deterministic automaton with $\Sigma_{\mathrm{nr}} \mathrm{SCC}$ automaton $G_{\mathrm{nr}}=\left\langle\Sigma, Q_{\mathrm{nr}}, \rightarrow_{\mathrm{nr}}, \tilde{Q}^{\circ}\right\rangle$, and let $\Sigma=\Sigma_{\mathrm{r}} \dot{\cup} \Sigma_{\mathrm{nr}}$. The OP-Verifier $V_{G}$ for $G$ is

$$
V_{G}=\left\langle\Sigma, Q_{V}, \rightarrow_{V}, Q_{V}^{\circ}\right\rangle
$$

where

- $Q_{V}=\{P \subseteq Q / \stackrel{\mathrm{nr}}{\leftrightarrow}|1 \leq| P \mid \leq 2\} \cup\{\perp\}$.

The state set of the verifier consists of sets of $\Sigma_{\mathrm{nr}}$-SCCs of $G$ of cardinality one or two, i.e, a single $\Sigma_{\mathrm{nr}}$-SCC and pairs of $\Sigma_{n r}$-SCCs, plus the special state $\perp$.

- $\rightarrow{ }_{V}$ consists of the following transitions:

$$
\begin{aligned}
& \{[x],[y]\} \stackrel{\sigma}{\rightarrow}\left\{\left[x^{\prime}\right],\left[y^{\prime}\right]\right\} \text { if } \sigma \in \Sigma_{\mathrm{r}},[x] \stackrel{\sigma}{\rightarrow} \mathrm{nr}\left[x^{\prime}\right] \text {, and } \\
& {[y] \stackrel{\sigma}{\rightarrow} \mathrm{nr}\left[y^{\prime}\right] \text {; }} \\
& \{[x],[y]\} \stackrel{\sigma}{\rightarrow}\left\{\left[x^{\prime}\right],[y]\right\} \quad \text { if } \sigma \in \Sigma_{\mathrm{nr}} \text { and }[x] \stackrel{\sigma}{\rightarrow} \mathrm{nr}\left[x^{\prime}\right] \text {; } \\
& \{[x],[y]\} \stackrel{\sigma}{\rightarrow} \perp \\
& \text { if } \sigma \in \Sigma_{\mathrm{r}},[x] \stackrel{\sigma}{\rightarrow} \mathrm{nr},[y] \text { is } \\
& \text { terminal, and }[y] \stackrel{9}{\overbrace{\mathrm{nr}}} \text {. }
\end{aligned}
$$

- $Q_{V}^{\circ}=\left\{\left\{\left[x^{\circ}\right],\left[y^{\circ}\right]\right\} \mid x^{\circ}, y^{\circ} \in Q^{\circ}\right\}$.

The initial state set of the verifier contains all pairs of $\Sigma_{\mathrm{nr}}$-SCCs of initial states of $G$.

Example 2: The $\Sigma_{\mathrm{nr}}$-SCC automaton corresponding to $G$ in Example 1 is $G_{\mathrm{nr}}$ shown in Fig. 2. The $\Sigma_{\mathrm{nr}}-\mathrm{SCC}$ are $[0]=\{0\},[1]=\{1\}$ and $[2]=\{2,3\}$. Notice that $[2]$

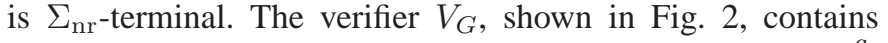
the following transitions: from $(7),\{[0]\}=\{[0],[0]\} \stackrel{a}{\rightarrow}$ $\{[1],[1]\}=\{[1]\},\{[0]\} \stackrel{\omega}{\rightarrow}\{[0]\},\{[1]\} \stackrel{b}{\rightarrow}\{[0]\}$, and $\{[2]\} \stackrel{c}{\rightarrow}\{[0]\}$; from (8), $\{[1]\} \stackrel{r}{\rightarrow}\{[1,2]\},\{[1,2]\} \stackrel{r}{\rightarrow}\{[1,2]\}$, $\{[1,2]\} \stackrel{r}{\rightarrow}\{[2]\},\{[1,2]\} \stackrel{y}{\rightarrow}\{[1,2]\},\{[2]\} \stackrel{r}{\rightarrow}\{[2]\}$, and $\{[2]\} \stackrel{y}{\rightarrow}\{[2]\}$; and, from (9), $\{[1],[2]\} \stackrel{b}{\rightarrow} \perp$, since [1] $\stackrel{b}{\rightarrow}$ nr [0], [2] is $\Sigma_{\mathrm{nr}}$-terminal, and [2] $\stackrel{b}{\rightarrow}$ nr does not hold. Note that state $\perp$ is reachable in $V_{G}$. It is shown below that this is a necessary and sufficient condition to confirm that $\theta(G)$ is not an OP-abstraction.

\section{Properties of the OP-Verifier}

This section establishes a key property of the OP-Verifier. The special state $\perp$ is reachable in the OP-Verifier if and only if the observer property is not satisfied. The main result in Theorem 3 depends on two lemmas to relate traces with the same projection to the states of the verifier: the OP-Verifier contains all pairs of $\Sigma_{n r}$-SCCs that can be reached by traces that project to the same relevant events.

Lemma 1: Let $V_{G}$ be the verifier for automaton $G$. Let $a, b \in \Sigma^{*}$ such that $\theta(a)=\theta(b)$ and $G \stackrel{a}{\rightarrow} x_{a}$ and $G \stackrel{b}{\rightarrow} x_{b}$. Then there exists $s \in \Sigma^{*}$ such that $\theta(a)=\theta(b)=\theta(s)$ and $V_{G} \stackrel{s}{\rightarrow}\left\{\left[x_{a}\right],\left[x_{b}\right]\right\}$. 
Proof: The claim is shown by induction on $n=|a|+|b|$.

In the base case, $n=0$ and thus $a=b=\varepsilon$. Then $x_{a}, x_{b} \in$ $Q^{\circ}$ and thus $\left\{\left[x_{a}\right],\left[x_{b}\right]\right\} \in Q_{V}^{\circ}$, i.e., $V_{G} \stackrel{\varepsilon}{\rightarrow}\left\{\left[x_{a}\right],\left[x_{b}\right]\right\}$.

Now assume the claim has been shown for all $a, b \in \Sigma^{*}$ such that $\theta(a)=\theta(b)$ and $|a|+|b| \leq n$. Consider $a, b \in \Sigma^{*}$ such that $\theta(a)=\theta(b)$ and $|a|+|b|=n+1$ and $G \stackrel{a}{\rightarrow} x_{a}$ and $G \stackrel{b}{\rightarrow} x_{b}$. As $\theta(a)=\theta(b)$ and $|a|+|b|>0$, either at least one of the traces $a$ or $b$ ends with an event in $\Sigma_{\mathrm{nr}}$, or both end with the same event in $\Sigma_{\mathrm{r}}$.

In the first case, assume without loss of generality $a=a^{\prime} \sigma$ for some $\sigma \in \Sigma_{\mathrm{nr}}$. Then $G \stackrel{a^{\prime}}{\rightarrow} x_{a}^{\prime} \stackrel{\sigma}{\rightarrow} x_{a}$ and $\theta\left(a^{\prime}\right)=\theta\left(a^{\prime} \sigma\right)=$ $\theta(a)=\theta(b)$, and by inductive assumption there exists $s \in \Sigma^{*}$ such that $\theta(s)=\theta\left(a^{\prime}\right)=\theta(b)$ and $V_{G} \stackrel{s}{\rightarrow}\left\{\left[x_{a}^{\prime}\right],\left[x_{b}\right]\right\}$. Given $x_{a}^{\prime} \stackrel{\sigma}{\rightarrow} x_{a}$ with $\sigma \in \Sigma_{\mathrm{nr}}$, it follows by construction of $V_{G}$ (8) that $V_{G} \stackrel{s}{\rightarrow}\left\{\left[x_{a}^{\prime}\right],\left[x_{b}\right]\right\} \stackrel{\sigma}{\rightarrow}\left\{\left[x_{a}\right],\left[x_{b}\right]\right\}$, i.e., $V_{G} \stackrel{s \sigma}{\rightarrow}\left\{\left[x_{a}\right],\left[x_{b}\right]\right\}$ with $\theta(s \sigma)=\theta(s)=\theta\left(a^{\prime}\right)=\theta(a)=\theta(b)$.

In the second case, $a=a^{\prime} \sigma$ and $b=b^{\prime} \sigma$ for some $\sigma \in \Sigma_{\mathrm{r}}$. Then $G \stackrel{a^{\prime}}{\rightarrow} x_{a}^{\prime} \stackrel{\sigma}{\rightarrow} x_{a}$ and $G \stackrel{b^{\prime}}{\rightarrow} x_{b}^{\prime} \stackrel{\sigma}{\rightarrow} x_{b}$ and $\theta\left(a^{\prime}\right)=\theta\left(b^{\prime}\right)$. By inductive assumption there exists $s^{\prime} \in \Sigma^{*}$ such that $\theta\left(s^{\prime}\right)=$ $\theta\left(a^{\prime}\right)=\theta\left(b^{\prime}\right)$ and $V_{G} \stackrel{s^{\prime}}{\rightarrow}\left\{\left[x_{a}^{\prime}\right],\left[x_{b}^{\prime}\right]\right\}$. Given $x_{a}^{\prime} \stackrel{\sigma}{\rightarrow} x_{a}$ and $x_{b}^{\prime} \stackrel{\sigma}{\rightarrow} x_{b}$ with $\sigma \in \Sigma_{\mathrm{r}}$, it follows by construction of $V_{G}$ (7) that $V_{G} \stackrel{s^{\prime}}{\rightarrow}\left\{\left[x_{a}^{\prime}\right],\left[x_{b}^{\prime}\right]\right\} \stackrel{\sigma}{\longrightarrow}\left\{\left[x_{a}\right],\left[x_{b}\right]\right\}$, i.e., $V_{G} \stackrel{s^{\prime} \sigma}{\longrightarrow}\left\{\left[x_{a}\right],\left[x_{b}\right]\right\}$ with $\theta\left(s^{\prime} \sigma\right)=\theta\left(a^{\prime} \sigma\right)=\theta(a)$ and $\theta\left(s^{\prime} \sigma\right)=\theta\left(b^{\prime} \sigma\right)=\theta(b)$.

Lemma 2: Let $V_{G}=\left\langle\Sigma, Q_{V}, \rightarrow_{V}, Q_{V}^{\circ}\right\rangle$ be the verifier for automaton $G$. Let $s \in \Sigma^{*}$ and $\{A, B\} \in Q_{V}$ such that $V_{G} \stackrel{s}{\rightarrow}$ $\{A, B\}$. Then there exist $a, b \in \Sigma^{*}$ such that $\theta(a)=\theta(b)=$ $\theta(s)$ and $G \stackrel{a}{\rightarrow} A$ and $G \stackrel{b}{\rightarrow} B$.

Proof: The claim is shown by induction on $n=|s|$.

In the base case, $n=0$ and thus $s=\varepsilon$. Note that $A, B \in$ $Q_{V}^{\circ}$. By Def. 5 there exist $x_{a}^{\circ} \in A$ such that $x_{a}^{\circ} \in Q^{\circ}$ and $x_{b}^{\circ} \in A$ such that $x_{b}^{\circ} \in Q^{\circ}$, which is enough to show $G \stackrel{\varepsilon}{\rightarrow} A$ and $G \stackrel{\varepsilon}{\rightarrow} B$.

Now consider $s=s^{\prime} \sigma$ such that $V_{G} \stackrel{s^{\prime}}{\rightarrow}\left\{A^{\prime}, B^{\prime}\right\} \stackrel{\sigma}{\rightarrow}\{A, B\}$, and assume by inductive assumption that there exist $a^{\prime}, b^{\prime} \in$ $\Sigma^{*}$ such that $\theta\left(a^{\prime}\right)=\theta\left(b^{\prime}\right)=\theta\left(s^{\prime}\right)$ and $G \stackrel{a^{\prime}}{\rightarrow} A^{\prime}$ and $G \stackrel{b^{\prime}}{\rightarrow} B^{\prime}$. Consider two cases.

If $\sigma \in \Sigma_{\mathrm{nr}}$, then by construction of $V_{G}$ (8), without loss of generality, there exist $x_{a}^{\prime} \in A^{\prime}$ and $x_{a} \in A$ such that $x_{a}^{\prime} \stackrel{\sigma}{\rightarrow} x_{a}$, and $B^{\prime}=B$. As $G \stackrel{a^{\prime}}{\rightarrow} A^{\prime}$, there exists $y_{a}^{\prime} \in A^{\prime}$ such that $G \stackrel{a^{\prime}}{\rightarrow} y_{a}^{\prime}$. Furthermore, $x_{a}^{\prime}, y_{a}^{\prime} \in A^{\prime}$ implies $x_{a}^{\prime} \stackrel{\mathrm{nr}}{\leftrightarrow} y_{a}^{\prime}$, i.e., there exists $t \in \Sigma_{\mathrm{nr}}^{*}$ such that $y_{a}^{\prime} \stackrel{t}{\rightarrow} x_{a}^{\prime}$. Thus $G \stackrel{a^{\prime}}{\rightarrow} y_{a}^{\prime} \stackrel{t}{\rightarrow} x_{a}^{\prime} \stackrel{\sigma}{\rightarrow}$ $x_{a} \in A$. It follows that $\theta\left(a^{\prime} t \sigma\right)=\theta\left(a^{\prime} \sigma\right)=\theta\left(s^{\prime} \sigma\right)=\theta(s)$, $\theta\left(b^{\prime}\right)=\theta\left(s^{\prime}\right)=\theta\left(s^{\prime} \sigma\right)=\theta(s), G \stackrel{a^{\prime} t \sigma}{\longrightarrow} A$, and $G \stackrel{b^{\prime}}{\rightarrow} B^{\prime}=B$.

If $\sigma \in \Sigma_{\mathrm{r}}$, then by construction of $V_{G}$ (7) there exist $x_{a}^{\prime} \in$ $A^{\prime}, x_{a} \in A, x_{b}^{\prime} \in B^{\prime}$, and $x_{b} \in B$ such that $x_{a}^{\prime} \stackrel{\sigma}{\rightarrow} x_{a}$ and $x_{b}^{\prime} \stackrel{\sigma}{\rightarrow} x_{b}$. As $G \stackrel{a^{\prime}}{\rightarrow} A^{\prime}$, there exists $y_{a}^{\prime} \in A^{\prime}$ such that $G \stackrel{a^{\prime}}{\rightarrow} y_{a}^{\prime}$. Furthermore, $x_{a}^{\prime}, y_{a}^{\prime} \in A^{\prime}$ implies $x_{a}^{\prime} \stackrel{\mathrm{nr}}{\leftrightarrow} y_{a}^{\prime}$, i.e., there exists $t_{a} \in \Sigma_{\mathrm{nr}}^{*}$ such that $y_{a}^{\prime} \stackrel{t_{a}}{\rightarrow} x_{a}^{\prime}$. Thus $G \stackrel{a^{\prime}}{\rightarrow} y_{a}^{\prime} \stackrel{t_{a}}{\rightarrow} x_{a}^{\prime} \stackrel{\sigma}{\rightarrow} x_{a} \in A$ and $\theta\left(a^{\prime} t_{a} \sigma\right)=\theta\left(a^{\prime} \sigma\right)=\theta\left(s^{\prime} \sigma\right)=\theta(s)$. Likewise, there exists $y_{b}^{\prime} \in B^{\prime}$ and $t_{b} \in \Sigma_{\mathrm{nr}}^{*}$ such that $G \stackrel{b^{\prime}}{\rightarrow} y_{b}^{\prime} \stackrel{t_{b}}{\rightarrow} x_{b}^{\prime} \stackrel{\sigma}{\rightarrow} x_{b} \in$ $B$ and $\theta\left(b^{\prime} t_{b} \sigma\right)=\theta\left(b^{\prime} \sigma\right)=\theta\left(s^{\prime} \sigma\right)=\theta(s)$.

Theorem 3: Let $G=\left\langle\Sigma, Q, \rightarrow, Q^{\circ}\right\rangle$ be a deterministic nonblocking automaton. The special state $\perp$ is reachable in $V_{G}$ if and only if $\theta(G)$ is not an OP-abstraction.
Proof: First assume that $\perp$ is reachable in $V_{G}$. By construction of $V_{G}(9)$, this means that there exists a reachable state $\{A, B\} \in Q_{V}$ such that $V_{G} \stackrel{s}{\rightarrow}\{A, B\} \stackrel{\sigma}{\rightarrow} \perp$, where $\sigma \in \Sigma_{\mathrm{r}}, x_{a} \stackrel{\sigma}{\rightarrow} y_{a}$ for some $x_{a} \in A$ and $y_{a} \in Q$, and $B$ is a terminal component such that $B \stackrel{\sigma}{\rightarrow}$ does not hold. By Lemma 2, there exists $a, b \in \Sigma^{*}$ such that $\theta(a)=\theta(b)=\theta(s)$ and $G \stackrel{a}{\rightarrow} A$ and $G \stackrel{b}{\rightarrow} B$. Then there exists $z_{a} \in A$ and $t_{a} \in \Sigma_{\mathrm{nr}}^{*}$ such that $G \stackrel{a}{\rightarrow} z_{a} \stackrel{t_{a}}{\rightarrow} x_{a} \stackrel{\sigma}{\rightarrow} y_{a}$, and since $G$ is nonblocking, there exists $u \in \Sigma^{*}$ such that $y_{a} \stackrel{u \omega}{\longrightarrow}$. Thus,

$$
G \stackrel{a}{\rightarrow} z_{a} \stackrel{t_{a}}{\rightarrow} x_{a} \stackrel{\sigma}{\rightarrow} y_{a} \stackrel{u \omega}{\longrightarrow} .
$$

Now let $s_{0}=b$ and $t_{0}=\sigma \theta(u)$. Then $\theta\left(s_{0}\right) t_{0}=\theta(b) \sigma \theta(u)=$ $\theta(a) \sigma \theta(u)=\theta\left(a t_{a} \sigma u\right) \in \theta\left(\mathcal{L}_{\mathrm{m}}(G)\right)$. However, there does not exist $t^{\prime} \in \Sigma^{*}$ such that $\theta\left(s_{0} t^{\prime}\right)=\theta\left(s_{0}\right) t_{0}$ and $s_{0} t^{\prime} \in \mathcal{L}_{\mathrm{m}}(G)$, because if such $t^{\prime}$ exists then $\theta\left(t^{\prime}\right)=t_{0}=\sigma \theta(u)$ and then as $s_{0}=b$ and $G \stackrel{b}{\rightarrow} B$ and $B$ is a terminal component, also $G \stackrel{s_{0}}{\rightarrow} B \stackrel{\sigma}{\rightarrow}$. However, $B$ was chosen such that $B \stackrel{\sigma}{\rightarrow}$ does not hold.

Conversely, assume $\theta(G)$ is not an OP-abstraction. Then there exist $s \in \mathcal{L}(G)$ and $t \in \Sigma_{\mathrm{r}}^{*}$ such that $\theta(s) t \in \theta\left(\mathcal{L}_{\mathrm{m}}(G)\right)$, and there does not exist $t^{\prime} \in \Sigma^{*}$ such that $\theta\left(s t^{\prime}\right)=\theta(s) t$ and $s t^{\prime} \in \mathcal{L}_{\mathrm{m}}(G)$. Let $u \leq t \omega$ be the longest prefix of $t \omega$ such that there exists $u^{\prime} \in \Sigma^{*}$ such that $\theta\left(u^{\prime}\right)=u$ and $s u^{\prime} \in \mathcal{L}(G)$. Clearly $u \neq t \omega$, since otherwise $t^{\prime}$ as above exists.

So let $t=u \sigma v$ with $\sigma \in \Sigma_{\mathrm{r}}$ and $v \in \Sigma_{\mathrm{r}}^{*}$. Then $\theta(s u) \sigma v=\theta(s) u \sigma v=\theta(s) t \in \theta\left(\mathcal{L}_{\mathrm{m}}(G)\right)$, so there exists $a \in \Sigma^{*}$ such that $\theta(a)=\theta(s u)$ and $G \stackrel{a}{\rightarrow} x_{a} \stackrel{\sigma}{\rightarrow}$. Since $s u^{\prime} \in \mathcal{L}(G)$, there exists $x_{b}^{\prime} \in Q$ such that $G \stackrel{s u^{\prime}}{\rightarrow} x_{b}^{\prime}$. By Remark 1, there exist $t_{b} \in \Sigma_{\mathrm{nr}}^{*}$ and $x_{b} \in Q$ such that $x_{b}^{\prime} \stackrel{t_{b}}{\rightarrow} x_{b}$ and $\left[x_{b}\right]$ is a terminal component. Let $b=s u^{\prime} t_{b}$. Note that $\theta(a)=\theta(s u)=\theta(s) u=\theta\left(s u^{\prime}\right)=\theta\left(s u^{\prime} t_{b}\right)=\theta(b)$ and $G \stackrel{a}{\rightarrow} x_{a}$ and $G \stackrel{b}{\rightarrow} x_{b}$. By Lemma 1 , there exists $s_{a b} \in \Sigma^{*}$ such that $\theta\left(s_{a b}\right)=\theta(a)=\theta(b)$ and $V_{G} \stackrel{s_{a b}}{\longrightarrow}\left\{\left[x_{a}\right],\left[x_{b}\right]\right\}$. Here it holds that $\left[x_{a}\right] \stackrel{\sigma}{\rightarrow}$ and $\left[x_{b}\right]$ is a terminal component and $\left[x_{b}\right] \stackrel{\sigma}{\rightarrow}$ does not hold, because otherwise $G \stackrel{b}{\rightarrow} x_{b} \stackrel{z \sigma}{\rightarrow}$ for some $z \in \Sigma_{\mathrm{nr}}^{*}$, and thus $b z \sigma=s u^{\prime} t_{b} z \sigma \in \mathcal{L}(G)$ in contradiction to the maximal choice of $u$ above. It follows by construction of $V_{G}(9)$ that $V_{G} \stackrel{s_{a b}}{\rightarrow}\left\{\left[x_{a}\right],\left[x_{b}\right]\right\} \stackrel{\sigma}{\rightarrow} \perp$.

Based on Theorem 3, the observer property can be checked by constructing the verifier automaton and checking whether it contains the state $\perp$.

\section{IMPLEMENTATION}

\section{A. The OP-Verifier Algorithm}

Algorithm 1 shows the pseudo-code of the OP-Verifier algorithm; this pseudo-code is the basis of the implementation of the OP-verifier algorithm within Supremica [22], which is further discussed in Section IV-C. The algorithm explores the state space of the verifier until a transition to $\perp$ is encountered, or until all possible verifier states have been constructed.

Verifier states are represented as ordered pairs $([x],[y])$ to represent a set $\{[x],[y]\} \in Q / \stackrel{\mathrm{nr}}{\leftrightarrow}$, with singletons $\{x\}$ represented as $([x],[x])$. To exploit the symmetry, all pairs are ordered such that $[x]<[y]$ based on a fixed but arbitrary ordering of the $\Sigma_{n r}$-SCC. The algorithm maintains the set 


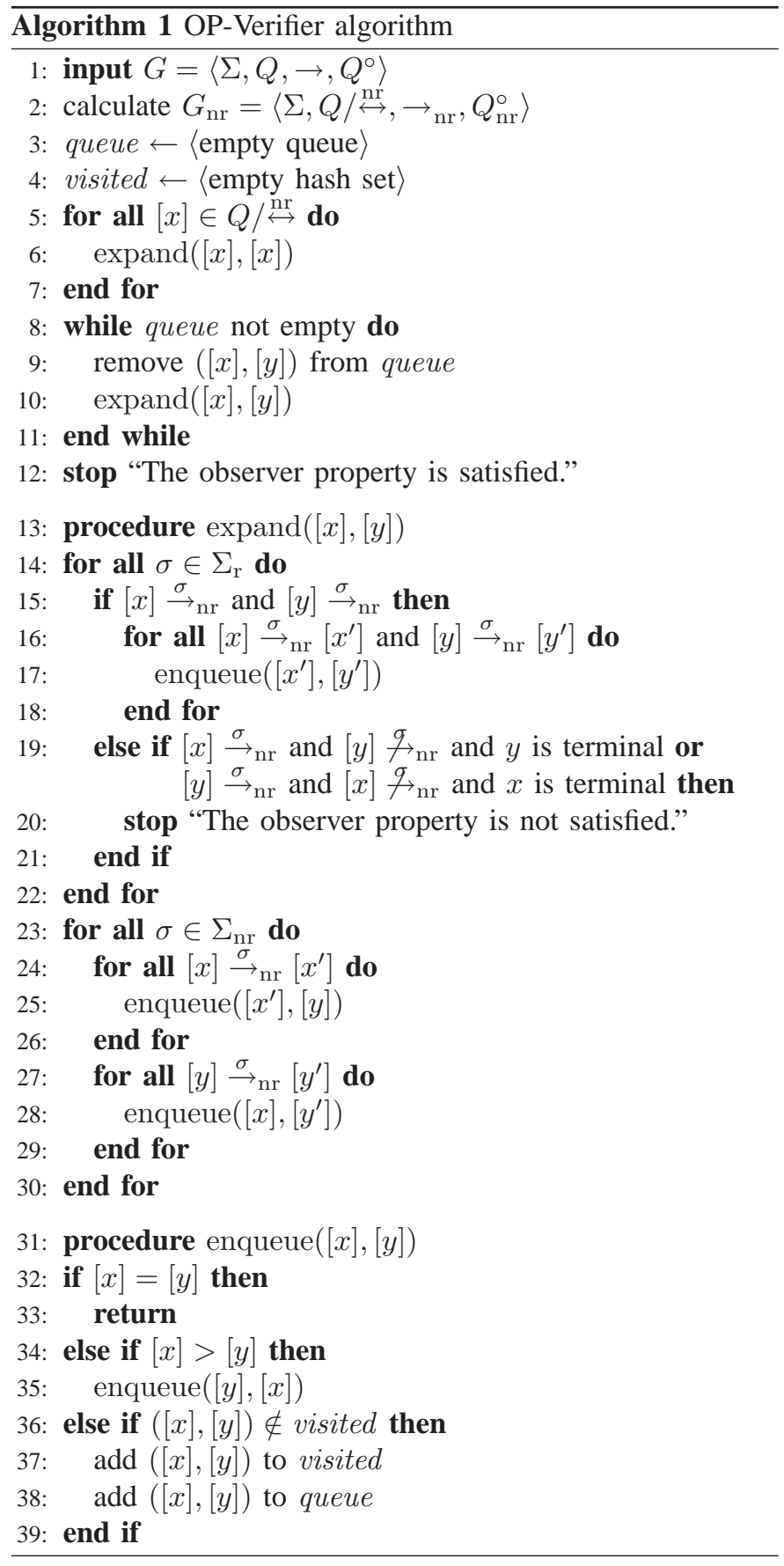

visited containing all pairs $([x],[y])$ discovered so far, and a queue containing those pairs that still need to be explored.

After construction of the $\Sigma_{\mathrm{nr}}$-SCC automaton using Tarjan's Algorithm [26], the loop in line 5 examines every $\Sigma_{n r}$ SCC $[x] \in Q / \stackrel{\text { nr }}{\leftrightarrow}$ and records the successors of the verifier state $\{[x]\}$ according to (7) and (8). This step assumes that all states are reachable. Afterwards, the loop in line 8 visits and expands all verifier states $\{[x],[y]\}$ resulting from the previous loop in line 5, and their successors.

Procedure expand checks for transitions originating from a verifier state $\{[x],[y]\}$. For relevant events, the loop in line 14 checks in line 15 for successor pairs according to (7), and then checks in line 19 whether condition (9) is satisfied. If so, the verifier clearly contains the state $\perp$, so the algorithm terminates and reports that the observer property is not satisfied. For non-relevant events, the loop in line 23 constructs successor pairs according to (8). Procedure enqueue adds new state pairs to the set visited and to the queue, ensuring the ordering and using the hash set visited to prevent any pairs from being processed more than once.

\section{B. Complexity}

The complexity of the OP-Verifier algorithm is determined by the complexity to construct the verifier. If the input is a deterministic automaton $G=\left\langle\Sigma, Q, \rightarrow, Q^{\circ}\right\rangle$ then the number of reachable states of $V_{G}$ is bounded by $|Q|^{2}+|Q|+1=$ $O\left(|Q|^{2}\right)$. To estimate the number of transitions of $V_{G}$, consider a transition $x \stackrel{\sigma}{\rightarrow} x^{\prime}$ in the input automaton $G$, and let $y \in Q$ be an arbitrary state. If $\sigma \in \Sigma_{\mathrm{r}}$, then this produces at most one transition $\{[x],[y]\} \stackrel{\sigma}{\rightarrow}\left\{\left[x^{\prime}\right],\left[y^{\prime}\right]\right\} \quad$ or $\{[x],[y]\} \stackrel{\sigma}{\rightarrow} \perp$ according to (7) or (9), and if $\sigma \in \Sigma_{\mathrm{nr}}$, then there is one transition $\{[x],[y]\} \stackrel{\sigma}{\rightarrow}\left\{\left[x^{\prime}\right],[y]\right\}$ according to (8). That is, every transition of $G$ produces up to $|Q|$ transitions in $V_{G}$. The deterministic automaton $G$ has up to $|\Sigma||Q|$ transitions, so the total number of transitions of $V_{G}$ is bounded by

$$
|\rightarrow||Q| \leq|\Sigma||Q|^{2}=O\left(|\Sigma||Q|^{2}\right) .
$$

Tarjan's algorithm to identify the $\Sigma_{\mathrm{nr}}$-SCC runs in $O(|\rightarrow|)=$ $O(|\Sigma||Q|)$ time [26], so it is dominated by the verifier construction. Therefore, (11) gives the worst-case time complexity of the OP-Verifier algorithm.

\section{Experimental Results}

The OP-Verifier algorithm has been implemented in Java and integrated in the discrete event systems tool Supremica [22]. Table I shows some experimental results to demonstrate the performance of the implementation. All experiments were run on a standard desktop computer using a single core $2.33 \mathrm{GHz} \mathrm{CPU}$ and $3 \mathrm{~GB}$ of RAM.

The test suite consists of 23 automata obtained as intermediate results during compositional nonblocking verification [9], and variations of such automata. The table shows for each automaton that was checked, the number of states $|Q|$, the number of events $|\Sigma|$, the total number of transitions $|\rightarrow|$, and the number of non-relevant transitions $|\stackrel{\mathrm{nr}}{\rightarrow}|$. Then the table shows the number of states $\left|Q_{V}\right|$ constructed by the OPVerifier algorithm, and the time taken to check the observer property. Furthermore, the time taken by Supremica [22] to compute a coarsest observation equivalence relation using the method in [27] is shown. This is the crucial step of the observer property verification algorithm proposed in [17] and is indicative of its performance. Finally, the table shows the verdict whether or not the given automaton satisfies the observer property.

Table I shows that the OP-Verifier algorithm can easily check automata with more than 100,000 states in a few seconds. With one exception, the number of verifier states is of the same order of magnitude as the number of states of the automaton, and the OP-Verifier algorithm runs significantly faster than observation equivalence. This is particularly true 
TABLE I

EXPERIMENTAL RESULTS

\begin{tabular}{|c|c|c|c|c|c|c|c|}
\hline \multicolumn{4}{|c|}{ Automaton } & \multicolumn{2}{|c|}{ OP-Verifier } & \multirow{2}{*}{$\begin{array}{c}\text { Obseq. } \\
\text { Time }\end{array}$} & \multirow[b]{2}{*}{ Verdict } \\
\hline$|Q|$ & $|\Sigma|$ & $|\rightarrow|$ & $|\stackrel{\mathrm{nr}}{\longrightarrow}|$ & $\left|Q_{V}\right|$ & Time & & \\
\hline 14934 & 38 & 118793 & 16087 & 31023 & $0.01 \mathrm{~s}$ & $0.21 \mathrm{~s}$ & false \\
\hline 14934 & 8985 & 118793 & 1272 & 16207 & $0.01 \mathrm{~s}$ & $0.09 \mathrm{~s}$ & alse \\
\hline 18816 & 42 & 532812 & 82656 & 101484 & $0.05 \mathrm{~s}$ & $7.29 \mathrm{~s}$ & false \\
\hline 19538 & 38 & 115369 & 41105 & 60807 & $0.02 \mathrm{~s}$ & $0.94 \mathrm{~s}$ & lse \\
\hline 19538 & 6028 & 114952 & 23616 & 43373 & $0.02 \mathrm{~s}$ & $0.73 \mathrm{~s}$ & false \\
\hline 21867 & 30 & 67058 & 34661 & 56530 & $0.02 \mathrm{~s}$ & $0.23 \mathrm{~s}$ & false \\
\hline 21867 & 16777 & 67058 & 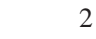 & 21870 & $0.01 \mathrm{~s}$ & $0.08 \mathrm{~s}$ & true \\
\hline 22599 & 22 & 156361 & 35667 & 58287 & $0.02 \mathrm{~s}$ & $1.04 \mathrm{~s}$ & false \\
\hline 22599 & 19062 & 156361 & 2187 & 24786 & $0.02 \mathrm{~s}$ & $0.14 \mathrm{~s}$ & true \\
\hline 22634 & 26 & 67100 & 35655 & 58291 & $0.02 \mathrm{~s}$ & $0.32 \mathrm{~s}$ & false \\
\hline 22634 & 9462 & 67100 & 2110 & 26030 & $0.01 \mathrm{~s}$ & $0.11 \mathrm{~s}$ & true \\
\hline 23313 & 40 & 232014 & 23784 & 47099 & $0.02 \mathrm{~s}$ & $0.49 \mathrm{~s}$ & false \\
\hline 23313 & 14366 & 232014 & 648 & 23961 & $0.02 \mathrm{~s}$ & $0.22 \mathrm{~s}$ & true \\
\hline 24938 & 32 & 75399 & 39305 & 64245 & $0.02 \mathrm{~s}$ & $0.27 \mathrm{~s}$ & false \\
\hline 24938 & 16265 & 75399 & 1932 & 27676 & $0.01 \mathrm{~s}$ & $0.09 \mathrm{~s}$ & true \\
\hline 31216 & 40 & 278251 & 33122 & 64340 & $0.03 \mathrm{~s}$ & $0.57 \mathrm{~s}$ & false \\
\hline 31216 & 14139 & 278251 & 4614 & 36750 & $0.03 \mathrm{~s}$ & $0.25 \mathrm{~s}$ & true \\
\hline 36277 & 18 & 288995 & 54049 & 90928 & $0.04 \mathrm{~s}$ & $3.38 \mathrm{~s}$ & false \\
\hline 36277 & 34013 & 288995 & 4926 & 41205 & $0.02 \mathrm{~s}$ & $0.27 \mathrm{~s}$ & false \\
\hline 49152 & 44 & 1546504 & 319488 & 368663 & $0.18 \mathrm{~s}$ & $93.89 \mathrm{~s}$ & false \\
\hline 49152 & 434 & 1446152 & 122880 & 5147232 & $27.71 \mathrm{~s}$ & $2.17 \mathrm{~s}$ & true \\
\hline 105619 & 20 & 680591 & 326173 & 1231667 & $1.01 \mathrm{~s}$ & $18.38 \mathrm{~s}$ & false \\
\hline 136656 & 36 & 1273580 & 249928 & 387611 & $0.17 \mathrm{~s}$ & $9.37 \mathrm{~s}$ & false \\
\hline
\end{tabular}

when the observer property is not satisfied, because the OPVerifier algorithm can terminate early as soon as the state $\perp$ is encountered during construction of the verifier. The case where the OP-Verifier is slower than observation equivalence has the largest number of non-relevant transitions among the examples that satisfy the observer property, while the observation equivalence algorithm quickly finds a good partition in this case. In all other cases, the OP-Verifier algorithm gives an answer in less than two seconds.

\section{CONCLUSIONS}

The OP-Verifier algorithm presented in this paper allows to efficiently check whether an abstraction obtained by a natural projection has the observer property. The procedure is a modified version of a previous one [11], which removes a restriction on the existence of cycles of non-relevant events while still ensuring quadratic complexity in the number of states. The new version of the verifier first merges all states connected by cycles of non-relevant events. The resulting (non-deterministic) automaton is then translated into a transition structure, in which the observer property is checked by verifying the reachability of a specific state. We are currently investigating how the $O P$-Verifier can be used to improve the OP-Search algorithm [19] in order to help computing reduced OP-abstractions.

\section{REFERENCES}

[1] C. G. Cassandras and S. Lafortune, Introduction to Discrete Event Systems, 2nd ed. Springer, 2008.

[2] R. C. Hill and D. M. Tilbury, "Modular Supervisory Control of Discrete Event Systems with Abstraction and Incremental Hierarchical Construction," in 8th Int. Workshop on Discrete Event Systems, WODES '06, Ann Arbor, MI, USA, July 2006, pp. 399-406.

[3] A. E. C. da Cunha and J. E. R. Cury, "Hierarchical Supervisory Control Based on Discrete Event Systems with Flexible Marking," IEEE Trans. Autom. Control, vol. 52, no. 12, pp. 2242-2253, Dec. 2007.
[4] L. Feng and W. M. Wonham, "Supervisory control architecture for discrete-event systems," IEEE Trans. Autom. Control, vol. 53, no. 6, pp. 1449-1461, July 2008.

[5] K. Schmidt, T. Moor, and S. Perk., "Nonblocking hierarchical control of decentralized discrete event systems," IEEE Trans. Autom. Control, vol. 53, no. 10, pp. 2252-2265, Nov. 2008.

[6] K. Schmidt and C. Breindl, "Maximally Permissive Hierarchical Control of Decentralized Discrete Event Systems," IEEE Trans. Autom. Control, vol. 56, no. 4, pp. 723-737, April 2011.

[7] K. Schmidt, H. Marchand, and B. Gaudin, "Modular and Decentralizd Supervisory Control of Concurrent Discrete Event Systems Using Reduced Systems Models," in 8th Int. Workshop on Discrete Event Systems, WODES '06, Ann Arbor, MI, USA, July 2006, pp. 149-154.

[8] L. Feng and W. M. Wonham, "Computationally Efficient Supervisor Design: Abstraction and Modularity," in 8th Int. Workshop on Discrete Event Systems, WODES'06, Ann Arbor, MI, USA, July 2006, pp. 3-8.

[9] H. Flordal and R. Malik, "Compositional verification in supervisory control," SIAM J. Control and Optimization, vol. 48, no. 3, pp. 19141938, 2009.

[10] P. N. Pena, J. E. R. Cury, and S. Lafortune, "Verification of Nonconflict of Supervisors Using Abstractions," IEEE Trans. Autom. Control, vol. 54, no. 12, pp. 2803-2815, 2009.

[11] _ - "Polynomial-Time Verification of the Observer Property in Abstractions," in 2008 American Control Conference, ACC'08, Seattle, USA, June 2008, pp. 465-470.

[12] K. C. Wong and W. M. Wonham, "Hierarchical Control of DiscreteEvent Systems," Discrete Event Dynamic Systems: Theory and Applications, vol. 6, no. 3, pp. 241-273, 1996.

[13] L. Feng and W. M. Wonham, "On the computation of natural observers in discrete-event systems," Discrete Event Dynamic Systems, vol. 20, no. 1, pp. 63-102, 2010.

[14] K. C. Wong, J. G. Thistle, R. P. Malhamé, and H.-H. Hoang, "Supervisory Control of Distributed Systems: Conflict Resolution," Discrete Event Dynamic Systems: Theory and Applications, vol. 10, pp. 131$186,2000$.

[15] K. Schmidt and C. Breindl, "On maximal permissiveness of hierarchical and modular supervisory control approaches for discrete event systems," in 9th Int. Workshop on Discrete Event Systems, WODES '08, Göteborg, Sweden, 2008, pp. 462-467.

[16] T. Yoo and S. Lafortune, "Polynomial-time verification of diagnosability of partially observed discrete-event systems," IEEE Trans. Autom. Control, vol. 47, no. 9, pp. 1491- 1495, Sep. 2002.

[17] K. C. Wong and W. M. Wonham, "On the Computation of Observers in Discrete-Event Systems," Discrete Event Dynamical Systems, vol. 14, no. 1, pp. 55-107, Jan. 2004.

[18] S. Jiang, R. Kumar, and H. E. Garcia, "Optimal sensor selection for discrete-event systems with partial observation," IEEE Trans. Autom. Control, vol. 48, no. 3, pp. 369-381, March 2003.

[19] P. N. Pena, J. E. R. Cury, R. Malik, and S. Lafortune, "Efficient Computation of Observer Projections using OP-Verifiers," in 10th Int. Workshop on Discrete Event Systems, WODES'10, Berlin, Germany, Aug. 2010, pp. 416-421.

[20] R. Kumar and P. N. Pena, "Private communication," Oct. 2012.

[21] H. J. Bravo, A. E. C. da Cunha, P. Pena, R. Malik, and J. E. R. Cury, "Generalised verification of the observer property in discrete event systems," in 11th Int. Workshop of Discrete Event Systems, WODES '12, Guadalajara, Mexico, 2012, pp. 337-342.

[22] K. Åkesson, M. Fabian, H. Flordal, and R. Malik, "Supremica - An integrated environment for verification, synthesis and simulation of discrete event systems," in 8th Int. Workshop of Discrete Event Systems, WODES '06, Ann Arbor, MI, USA, 2006, pp. 384-385.

[23] J. E. Hopcroft, R. Motwani, and J. D. Ullman, Introduction to Automata Theory, Languages, and Computation. Addison-Wesley, 2001.

[24] H. Zhong and W. M. Wonham, "On the Consistency of Hierarchical Supervision in Discrete-Event Systems," IEEE Trans. Autom. Control, vol. 35, no. 10, pp. 1125-1134, Oct. 1990.

[25] L. R. Foulds, Graph theory applications. Universitext, 1992.

[26] R. Tarjan, "Depth first search and linear graph algorithms," SIAM J. Comput., vol. 1, no. 2, pp. 146-160, Jun. 1972.

[27] T. Bolognesi and S. A. Smolka, "Fundamental results for the verification of observational equivalence: a survey," in Protocol Specification, Testing and Verification VII: IFIP WG6.1 7th Int. Conf. on Protocol Specification, Testing and Verification, H. Rudin and C. H. West, Eds. Amsterdam, The Netherlands: North-Holland, 1987, pp. 165-179. 